\title{
Supplementary figure.
}

MIF inhibitors used in this study: 1: $c 1(\mathrm{O}) \operatorname{cc} 2 \mathrm{c}(\mathrm{cc} 1) \mathrm{c}(\mathrm{c}(\mathrm{c} 1 \mathrm{cc}(\mathrm{c}(\mathrm{cc} 1) \mathrm{O}) \mathrm{O}) \operatorname{co} 2)=\mathrm{O} . \mathbf{2}$ :

c1(CSc2ecncc2) $\operatorname{cc}(\mathrm{c}(\mathrm{O}) \operatorname{cc} 1) \mathrm{O} . \quad$ 3: $\quad \mathrm{c} 1(\mathrm{O}) \operatorname{cc} 2 \mathrm{c}(\mathrm{cc} 1) \operatorname{cc}(\mathrm{c} 1 \operatorname{ccccc} 1) \mathrm{c}(\mathrm{o} 2)=\mathrm{O} . \quad \mathbf{4}$

$\mathrm{c} 1(\mathrm{O}) \operatorname{cc} 2 \mathrm{c}(\mathrm{cc} 1) \operatorname{cc}(\mathrm{C}(\mathrm{S})=[\mathrm{NH} 2]) \mathrm{c}(\mathrm{o} 2)=\mathrm{O}$.

c12[C@@H]3[C@H]([C@@H](C(=O)O)Nc1ccc(c2)Br)CC=C3.

$\operatorname{c1}(\mathrm{O}) \operatorname{cc} 2 \mathrm{c}(\mathrm{cc} 1) \operatorname{cc}(\mathrm{C}(=\mathrm{O}) \mathrm{C}) \mathrm{c}(\mathrm{o} 2)=\mathrm{O}$

$\mathrm{COC}(=\mathrm{O}) \mathrm{C}[\mathrm{C} @ @ \mathrm{H}] 1 \mathrm{O}[\mathrm{NH}]=\mathrm{C}(\mathrm{C} 1) \mathrm{c} 1 \mathrm{ccc}(\mathrm{O}) \mathrm{cc} 1 . \quad 8$ :

$\operatorname{CCOC}(=\mathrm{O}) \operatorname{c} 1 \operatorname{cc} 2 \operatorname{ccc}(\operatorname{cc} 20 \mathrm{oc} 1=\mathrm{O}) \mathrm{O}$

c12c([C@@H](C(=O)O)C[C@H]1c1cccs1)cc(CCc1c3c(ccc1)cccs3)c(c2)NC(C(C)C)

$=0 . \quad$ 10: $\quad \mathrm{C}(\operatorname{clccc}(\mathrm{Cl}) \operatorname{cc} 1)(\operatorname{c} 1 \operatorname{ccc}(\mathrm{Cl}) \operatorname{cc} 1)(\operatorname{clccc}(\mathrm{Cl}) \operatorname{cc} 1) \mathrm{CC}(=\mathrm{O}) \mathrm{O} . \quad$ 11:

$\mathrm{c} 1(\mathrm{CCCC} 2 \mathrm{CCCCC} 2) \mathrm{oc}(\mathrm{C}(=\mathrm{O}) \mathrm{N} 2 \mathrm{CCN}(\mathrm{CC} 2) \mathrm{Cc} 2 \operatorname{ccccc} 2) \mathrm{c}(\mathrm{C}) \mathrm{n} 1$.

$\mathrm{O} \mathrm{IC}(=\mathrm{Clc} 1 \operatorname{ccc}(\operatorname{cc} 1) \mathrm{O}) \mathrm{C}(\mathrm{O})=\mathrm{O} . \quad$ 13: $\quad$ D-dopachrome. 14: 5,6-dihydroxyindole-2carboxylic acid (DHICA)

HIV protease-1 inhibitors used in this study (PDB code in parentheses is the complex structure from which the compound originated): compound 29: $\mathrm{C} 1(\mathrm{c} 2 \mathrm{ccc}(\mathrm{F}) \mathrm{cc} 2)(\mathrm{SCCS} 1) \mathrm{CCCN} 3 \mathrm{CCC}(\mathrm{c} 4 \mathrm{ccc}(\mathrm{Cl}) \mathrm{cc} 4)(\mathrm{O}) \mathrm{CC} 3$ (1aid), compound 30: $\operatorname{c1}(\mathrm{OCC} 2 \mathrm{~N}(\mathrm{~S}(\mathrm{~N}(\mathrm{C}(\mathrm{C}(\mathrm{C} 2 \mathrm{O}) \mathrm{O}) \mathrm{COc} 3 \operatorname{cccc} 3) \mathrm{Cc} 4 \operatorname{cccc} 4)(=\mathrm{O})=\mathrm{O}) \mathrm{Cc} 5 \operatorname{ccccc} 5) \operatorname{cccc} 1$

(1ajv), compound 31: [4r-(4alpha,5alpha,6beta,7beta)]-3,3'-[[tetrahydro-5 6-dihydroxy- 
2-oxo-4,7-bis( phenylmethyl)-1h-1,3 diazepine-1,3(2h)-diyl] bis(methylene)]bis[N-2

thiazolylbenzamide

(1bv7),

compound

32:

$\mathrm{C}(\mathrm{N}(\mathrm{Cc} 1 \mathrm{ncccc} 1) \mathrm{C})(=\mathrm{O}) \mathrm{NC}(\mathrm{C}(=\mathrm{O}) \mathrm{NC}(\mathrm{C}(\mathrm{C}(\mathrm{C}(\mathrm{NC}(=\mathrm{O}) \mathrm{C}(\mathrm{C}(\mathrm{C}) \mathrm{C}) \mathrm{NC}(\mathrm{N}(\mathrm{Cc} 2 \mathrm{ncccc} 2) \mathrm{C})$

$=\mathrm{O}) \mathrm{Cc} 3 \operatorname{ccccc} 3)(\mathrm{O}) \mathrm{O})(\mathrm{F}) \mathrm{F}) \mathrm{Cc} 4 \operatorname{cccc} 4) \mathrm{C}(\mathrm{C}) \mathrm{C} \quad$ (1dif), compound

33:

$\mathrm{C}(\mathrm{N} 1 \mathrm{C}(\mathrm{C}(=\mathrm{O}) \mathrm{NC}(\mathrm{C})(\mathrm{C}) \mathrm{C}) \mathrm{CSC} 1)(=\mathrm{O}) \mathrm{C}(\mathrm{C}(\mathrm{NC}(=\mathrm{O}) \mathrm{C}(\mathrm{NC}(=\mathrm{O}) \mathrm{COc} 2[\mathrm{c}] 3[\mathrm{c}](\operatorname{cncc} 3) \mathrm{ccc}$

2)(CSC)(c4 $4 \operatorname{cccc} 4) \mathrm{O}$

(1hpx),

compound

34:

$\mathrm{C}(=\mathrm{O})(\mathrm{C}(\mathrm{NC}(=\mathrm{O}) \mathrm{C}(\mathrm{CC}(\mathrm{C}) \mathrm{C}) \mathrm{N}) \mathrm{CCC}(=\mathrm{O}) \mathrm{N}) \mathrm{NC}(\mathrm{C}(=\mathrm{O}) \mathrm{NC}(\mathrm{C}(=\mathrm{O}) \mathrm{O}) \mathrm{CO}) \mathrm{CCC}(=\mathrm{O}) \mathrm{O}$

(1hte),

compound

35:

$\mathrm{C}(=\mathrm{O})(\mathrm{C} 1 \mathrm{C}(\mathrm{SC}(\mathrm{C}(\mathrm{C}(=\mathrm{O}) \mathrm{NCc} 2 \operatorname{cccc} 2) \mathrm{NC}(=\mathrm{O}) \mathrm{Cc} 3 \operatorname{cccc} 3) \mathrm{N} 1)(\mathrm{C}) \mathrm{C}) \mathrm{NC}(\mathrm{Cc} 4 \operatorname{ccccc} 4) \mathrm{C}$

$\mathrm{O}$

(1htf),

compound

36:

$\operatorname{c1} 2 \mathrm{c}(\operatorname{cccc} 1) \mathrm{NC}(=\mathrm{N} 2) \mathrm{CNC}(=\mathrm{O}) \mathrm{CC}(\mathrm{C}(\mathrm{NC}(=\mathrm{O}) \mathrm{C} 3 \mathrm{C}(\mathrm{SC}(\mathrm{C}(\mathrm{C}(=\mathrm{O}) \mathrm{NCc} 4 \operatorname{ccccc} 4) \mathrm{NC}(=\mathrm{O})$

Cc5cccc5)N3)(C)C)Cc6cccc6)O (1htg), compound 37: 2-phosphoglycolic acid (1hvi),

compound

38:

$\mathrm{C} 1(\mathrm{~N}(\mathrm{C}(\mathrm{C}(\mathrm{C}(\mathrm{C}(\mathrm{N} 1 \mathrm{Cc} 2 \mathrm{c}[\mathrm{c}] 3[\mathrm{c}](\mathrm{cc} 2) \operatorname{ccc} 3) \mathrm{Cc} 4 \mathrm{ccccc} 4) \mathrm{O}) \mathrm{O}) \mathrm{Cc} 5 \mathrm{ccccc} 5) \mathrm{Cc} 6 \mathrm{c}[\mathrm{c}] 7[\mathrm{c}](\mathrm{cc}$

6) $\operatorname{cccc} 7)=\mathrm{O}(1 \mathrm{hvr})$, compound 39: 2-carbonylquinoline - phenylalaninol group decahydro-1-methylisoquinoline-2-carbonyl - tertiary-butylamino group (1hxb), compound 40: ritonavir (1hxw), compound 41: naphthyloxyacetyl - cyclohexyl alapsi(Choh-Choh)-Val -2-aminomethyl-pyridine (1ivp), compound 42: 2- 
carbonylquinoline - phenylalanylmethane -3-(carboxyamide (2-carboxyamide-2tertbutylethyl)) $\quad$ penta $\quad(1 \mathrm{jld}), \quad$ compound $\mathrm{C} 1(\mathrm{~N}(\mathrm{C}(\mathrm{C}(\mathrm{C}(\mathrm{C}(\mathrm{N} 1 \mathrm{Cc} 2 \operatorname{ccc}(\operatorname{cc} 2) \mathrm{CO}) \mathrm{Cc} 3 \operatorname{cccc} 3) \mathrm{O}) \mathrm{O}) \mathrm{Cc} 4 \operatorname{ccccc} 4) \mathrm{Cc} 5 \operatorname{ccc}(\operatorname{cc} 5) \mathrm{CO})=\mathrm{O}$ (1mes), compound 44: tertiary-butoxyformic acid - phenylalaninol group dimethylamine -phenylalaninol group - tertiary-butoxyformic acid (lodw), compound 45: (5r,6r)-2,4-bis-(4-hydroxy-3-methoxybenzyl)-1,5dibenzyl-3-oxo-6-hydroxy-1,2,4triazacycloheptane $\quad(1 \mathrm{pro}), \quad$ compound $\quad \mathbf{4 6}$ : $\mathrm{C} 1(\mathrm{C}(=\mathrm{C}(\mathrm{C}=\mathrm{C}(\mathrm{O} 1) \mathrm{C}(\mathrm{Cc} 2 \operatorname{ccccc} 2) \mathrm{CC}) \mathrm{O}) \mathrm{C}(\mathrm{c} 3 \operatorname{cc}(\operatorname{ccc} 3) \mathrm{NC}(=\mathrm{O}) \mathrm{CCNC}(=\mathrm{O}) \mathrm{OC}(\mathrm{C})(\mathrm{C}) \mathrm{C})$ $\mathrm{C} 4 \mathrm{CC} 4)=\mathrm{O}(2 \mathrm{upj})$, compound 47: N,N-bis- (2(R)-hydroxy-1 (S)-indanyl-2,6- $(\mathrm{R}, \mathrm{R})$ diphenylmethyl-4-hydroxy-1,7-heptandiamide (4hpv).

GST inhibitors used in this study (PDB code in parentheses is the complex structure from which the compound originated): compound 48: benzylcysteine - phenylglycine (10gs), compound 49: glutathione - [2,3-dichloro-4- (2-methylene-1-oxobutyl) phenoxyacetic acid (11gs), compound 50: S-nonyl-cysteine (12gs), compound 51: 1-(Sglutathionyl)-2,4-dinitrobenzene (18gs), compound 52: glutamyl group - S-(4bromobenzyl)cystine (1aqv), compound 53: glutamyl group - S-(2,3,6trinitrophenyl)cysteine (1aqx), compound 54: S-hexylglutathione (1pgt), compound 55: cibacron blue (20gs), compound 56: chlorambucil (21gs), compound 57: ethacrynic 
acid (2gss), compound $\quad 58: \quad$ (9r,10r)-9-(S-glutathionyl)-10-hydroxy-9,10 dihydrophenanthrene (2pgt), compound 59: 2-amino-4- $\quad[1-\quad$ (carboxymethylcarbamoyl)-2- (9-hydroxy-7 8-dioxo- 7,8,9,10- tetrahydro-benzo [def] chrysen-10 ylsulfanyl) -ethylcarbamoyl]-butyric acid (3pgt).

Thermolysin inhibitors used in this study (PDB code in parentheses is the complex structure from which the compound originated): compounds 60: aspartic acid, compound 61: aspartame, compound 62: phenyl alanine, compound 63: 1benzylsuccinate (1hyt), compound 64: phenylalanine phosphinic acid - deaminomethyl-phenylalanine (1os0), compound 65: (6-methyl-3,4-dihydro-2H-chromen-2-Yl) methylphosphonate (1pe5), compound 66: 2-(4-methylphenoxy) ethylphosphonate - 3methylbutan-1-amine (1pe7), compound 67: 2-ethoxyethylphosphonate - 3methylbutan-1-amine (1pe8), compound 68: (2-sulfanyl-3-phenylpropanoyl)-Phe-Tyr (1qf0), compound 69: [2(R,S)-2-sulfanylheptanoyl]-Phe-Ala (1qf1), compound 70: [(2S)-2-sulfanyl-3-phenylpropanoyl]-Gly-(5-phenylproline) (1qf2), compound 71: n-(1(2(R, S)-carboxy-4-phenylbutyl) cyclopentylcarbonyl)- (S)-tryptophan (1thl), compound 72: (R)-retrothiorphan (1z9g), compound 73: (S)-thiorphan (1zdp), compound 74: hydroxamic acid (4tln), compound 75: phenylalanine phosphinic acid (4tmn), compound 76: Honh-benzylmalonyl-L-alanylglycine-P-nitroanilide (5tln), 
compound 77: Cbz-Gly ${ }^{\mathrm{P}}-\mathrm{Leu}-\mathrm{Leu}\left(\mathrm{Zg}^{\mathrm{P}} \mathrm{Ll}\right)$ (5tmn), compound 78: Cbz-Gly ${ }^{\mathrm{P}}-(\mathrm{O})-\mathrm{Leu}-$ Leu $\left(\mathrm{Zg}^{\mathrm{P}}(\mathrm{O}) \mathrm{Ll}\right)(6 \mathrm{tmn})$, compound 79: $\mathrm{CH}_{2} \mathrm{CO}(\mathrm{N}-\mathrm{OH}) \mathrm{Leu}-\mathrm{OCH}_{3}(7 \mathrm{tln})$, compound 80: benzyloxycarbonyl-D-Ala (1kto), compound 81: benzyloxycarbonyl-L-Ala (1kl6), compound 82: benzyloxycarbonyl-D-Thr (1kro), compound 83: benzyloxycarbonyl-LThr (1kj0), compound 84: benzyloxycarbonyl-D-Asp (1ks7), compound 85: benzyloxycarbonyl-L-Asp (1kkk), compound 86: benzyloxycarbonyl-D-Glu (1kr6) and compound 87: benzyloxycarbonyl-L-Glu (1kjp) 


\section{FIGURE CAPTIONS}

Figure 1 MIF active compounds

Figure 2 COX-2 active compounds 15: Sc-558 (1-phenylsulfonamide-3trifluoromethyl-5-parabromophenylpyrazole). 16: diclofenac. 17: indomethacin. 18: arachidonic acid. 19: diflunisal. 20: etodolac. 21: ketoprofen. 22: naproxen. 23: nimesulide. 24: prostaglandin H2. 25: piroxicam. 26: rofecoxib. 27: sulindac. 28: suprofen.

Figure 3 HIV protease-1 active compounds 29: ligand of 1aid. 30: ligand of 1ajv. 31: ligand of 1bv7. 32: ligand of 1dif. 33: ligand of 1hpx. 34: ligand of 1hte. 35: ligand of 1htf. 36: ligand of 1htg. 37: ligand of 1hvi. 38: ligand of 1hvr. 39: ligand of 1hxb. 40: ligand of 1hxw. 41: ligand of 1ivp. 42: ligand of 1jld. 43: ligand of 1mes. 44: ligand of 1odw. 45: ligand of 1pro. 46: ligand of 2upj. 47: ligand of 4phv.

Figure 4 GST active compounds 48: ligand of 10gs. 49: ligand of 11gs. 50: ligand of 12gs. 51: ligand of 18gs. 52: ligand of 1aqv. 53: ligand of 1aqx. 54: ligand of 1pgt. 55: ligand of $20 \mathrm{gs}$. 56: ligand of $21 \mathrm{gs} .57$ : ligand of $2 \mathrm{gss} .58$ : ligand of 2 pgt. 59: ligand of 3pgt. 
Figure 5 Thermolysin active compounds 60: aspartic acid. 61: aspartame. 62: phenyl alanine. 63: ligand of 1hyt. 64: ligand of 1 os 0.65 : ligand of 1pe5. 66: ligand of 1 pe7. 67: ligand of 1pe8. 68: ligand of 1qf0. 69: ligand of 1qf1. 70: ligand of 1qf2. 71: ligand of 1thl. 72: ligand of 1z9g. 73: ligand of 1zdp. 74: ligand of 4tln. 75: ligand of 4tmn. 76: ligand of 5tln. 77: ligand of 5tmn. 78: ligand of 6tmn. 79: ligand of 7th. 80: ligand of benzyloxycarbonyl-D-Ala. 81: ligand of benzyloxycarbonyl-D-Asp. 82: ligand of benzyloxycarbonyl-D-Glu. 83: ligand of benzyloxycarbonyl-D-Thr. 84: ligand of benzyloxycarbonyl-L-Ala. 85: ligand of benzyloxycarbonyl-L-Asp. 86: ligand of benzyloxycarbonyl-L-Glu. 87: ligand of benzyloxycarbonyl-L-Thr. 
<smiles>O=c1c(-c2ccc(O)c(O)c2)coc2cc(O)ccc12</smiles><smiles>CCOC(=O)c1cc2ccc(O)cc2oc1=O</smiles><smiles>Oc1ccc(CSc2ccncc2)cc1O</smiles><smiles>CC(C)C(=O)Nc1cc2c(cc1CCc1cccc3ccccc13)C(C(=O)O)CC2c1cccs1</smiles><smiles>C/C=C/c1cc2ccc(O)cc2oc1=O</smiles><smiles>O=C(O)CC(c1ccc(Cl)cc1)(c1ccc(Cl)cc1)c1ccc(Cl)cc1</smiles><smiles>O=C(O)C1Nc2ccc(Br)cc2C2C=CCC12</smiles><smiles>O=C(c1cnc(CCCC2CCCCC2)o1)N1CCN(Cc2ccccc2)CC1</smiles><smiles>CC(=O)c1cc2ccc(O)cc2oc1=O</smiles><smiles>O=C(O)/C(O)=C\c1ccc(O)cc1</smiles><smiles>COC(=O)Cc1cc(-c2ccc(O)cc2)no1</smiles><smiles>O=C([O-])c1cc2cc(O)c(O)cc2[nH]1</smiles> 
<smiles>O=C([O-])C1=CC2=CC(=O)C(O)=CC2=N1</smiles>

Figure 1 Y. Fukunishi, S. Kubota and H. Nakamura, "Noise reduction method for molecular interaction energy". 


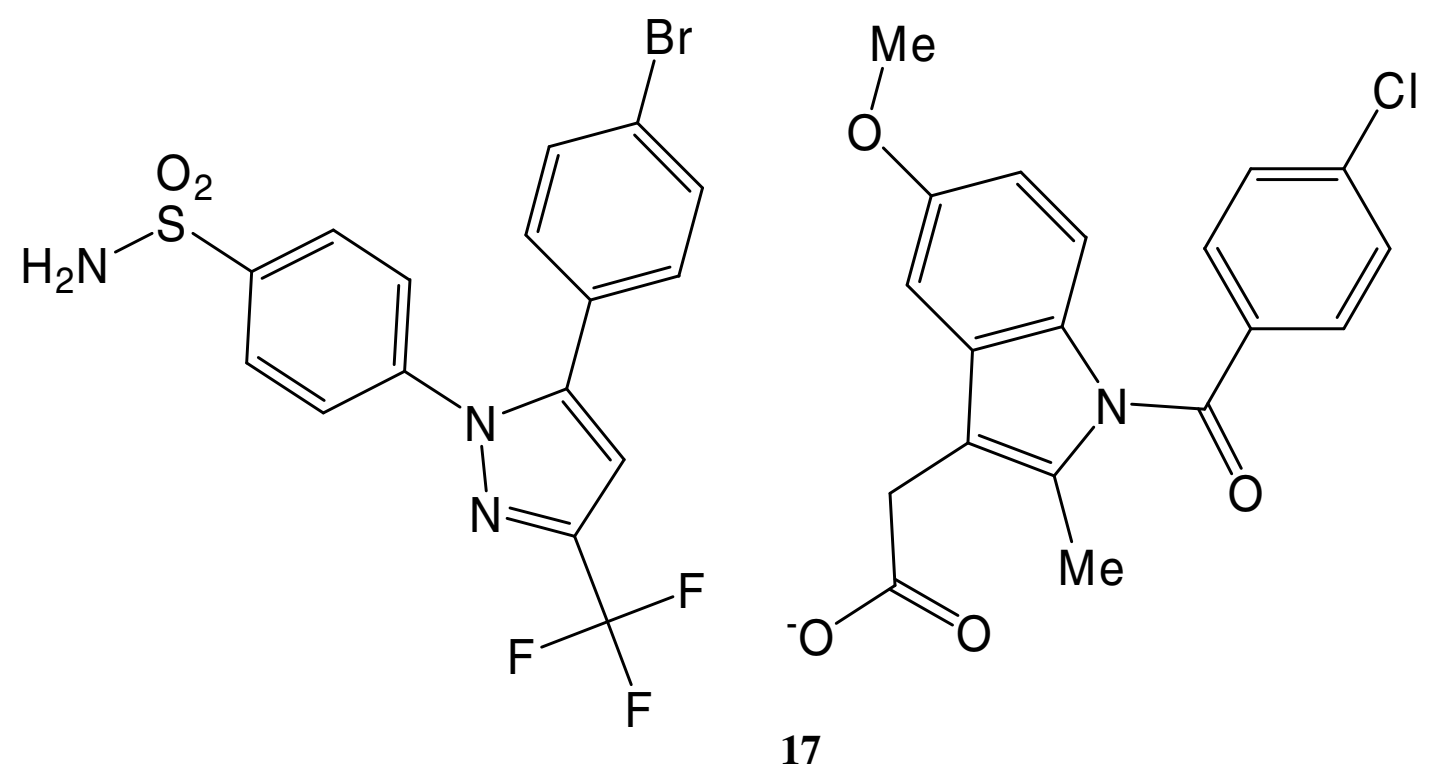

15

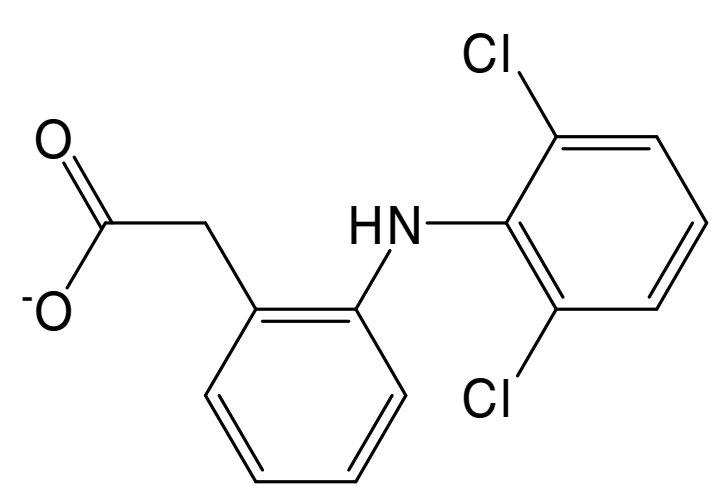

16

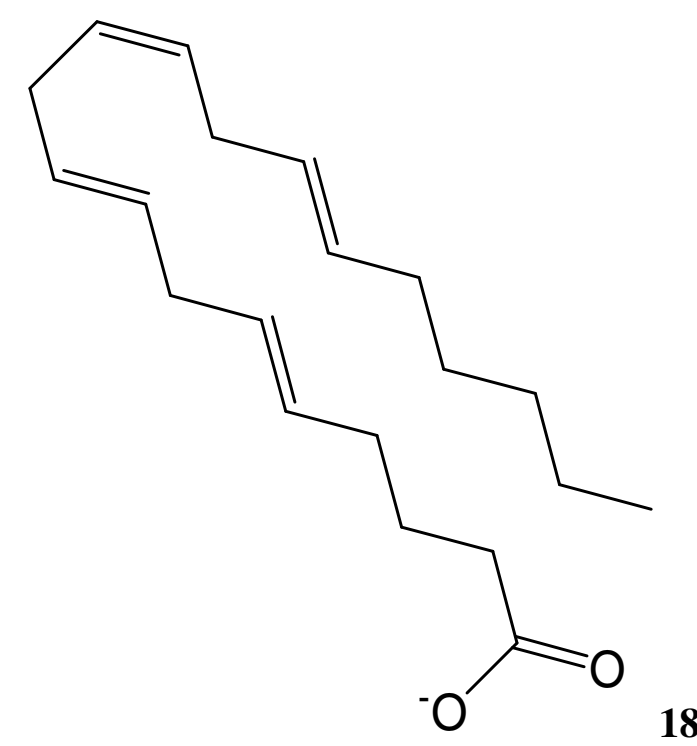




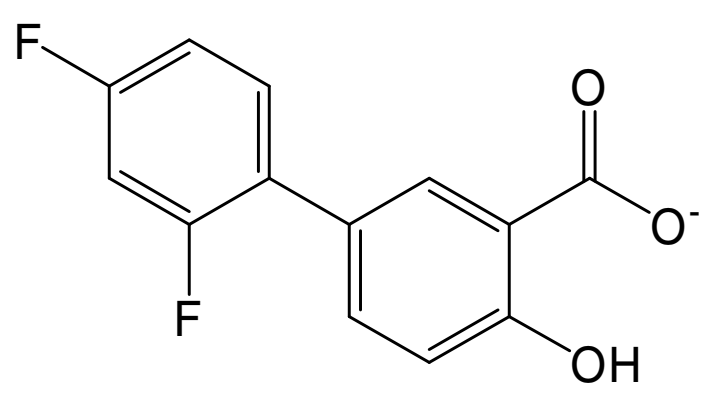

19

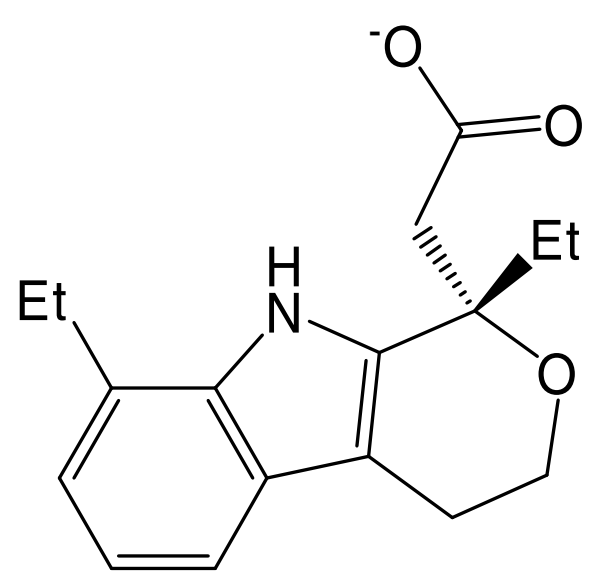

20

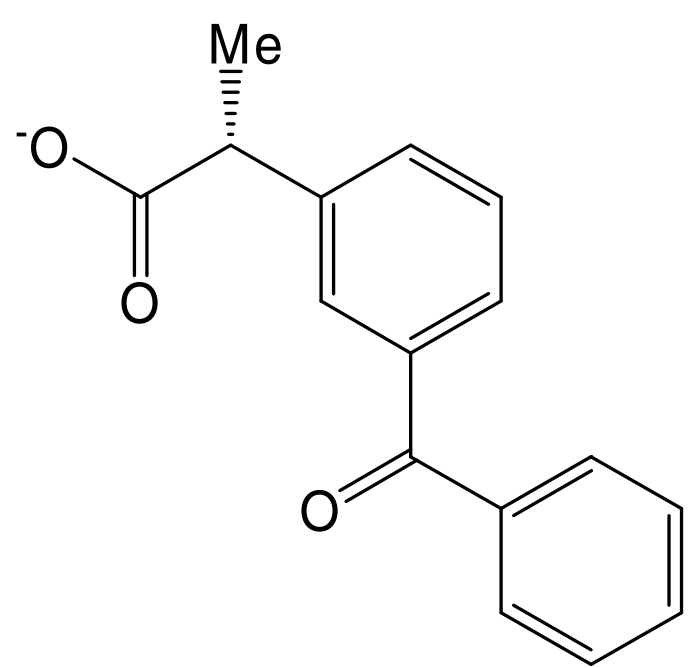

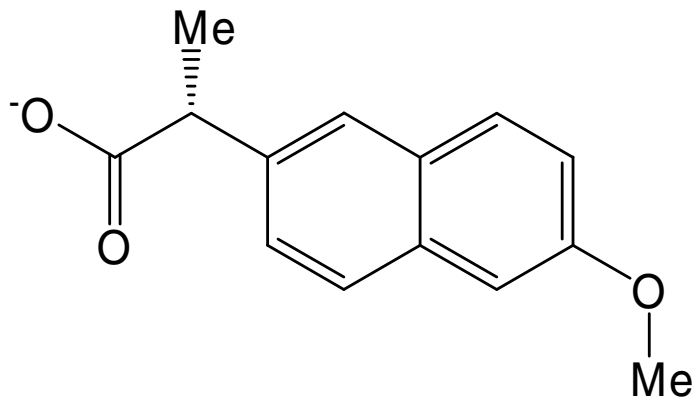

22

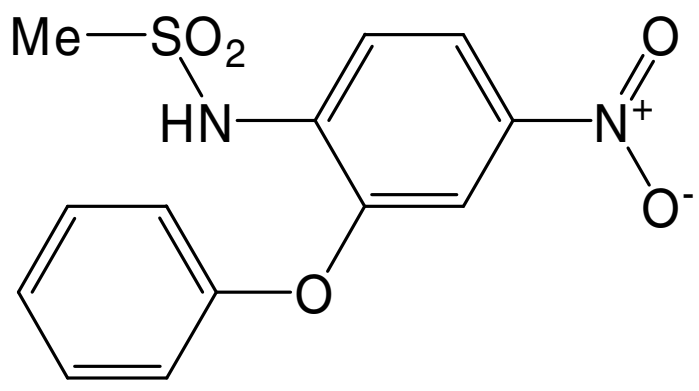

23

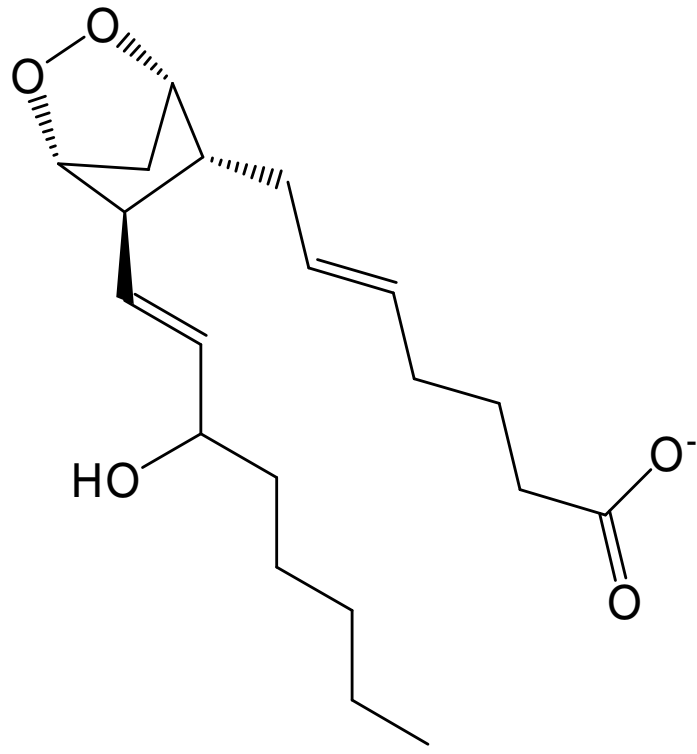

21 

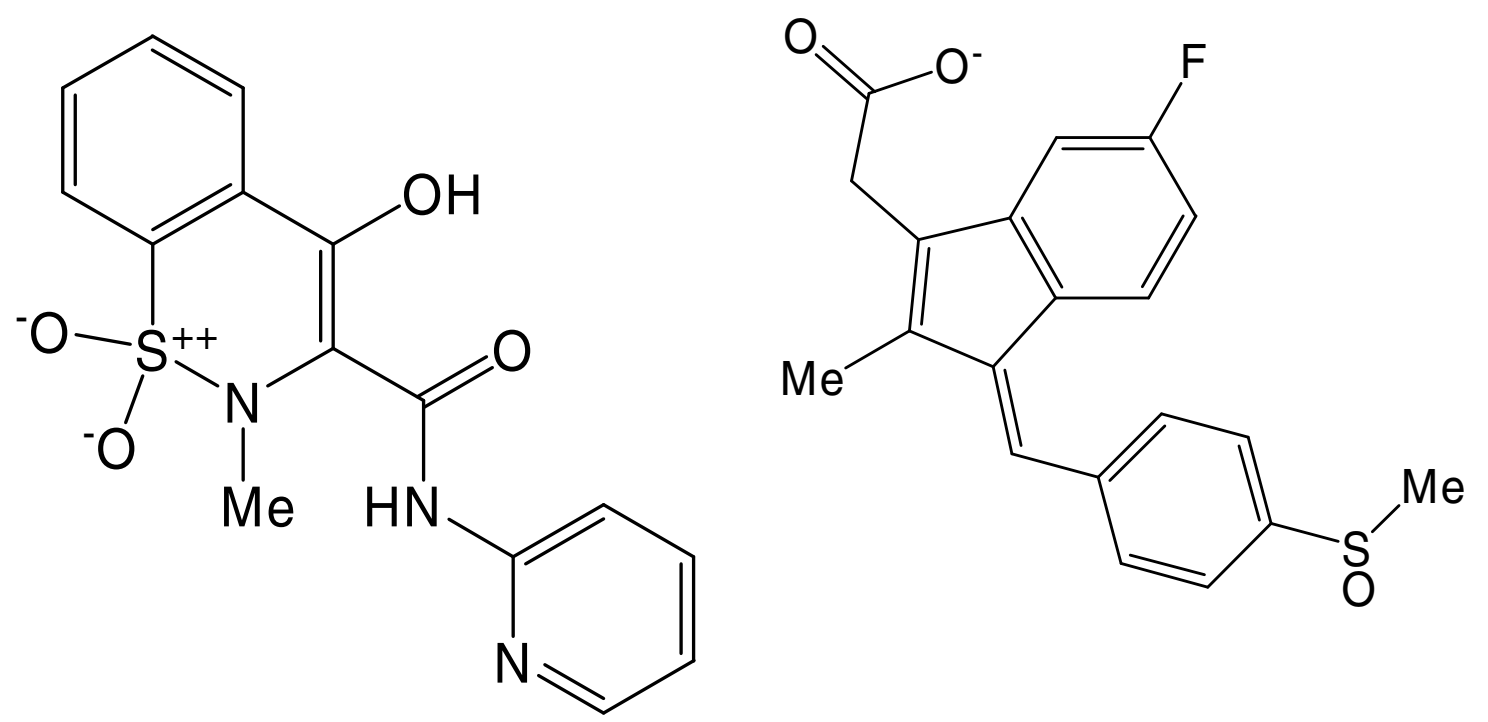

27

25
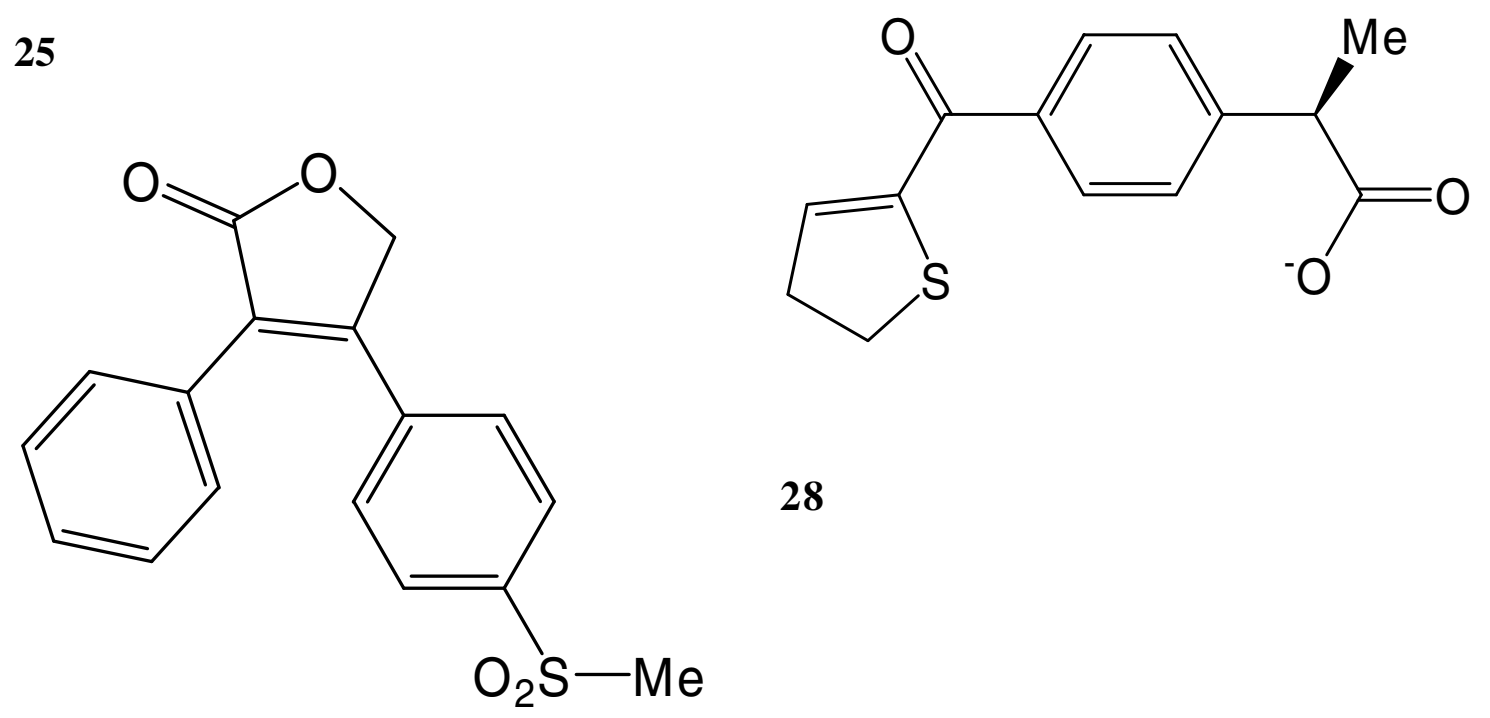

28

26

Figure 2 Y. Fukunishi, S. Kubota and H. Nakamendise reduction method for molecular interaction ene’rgy 


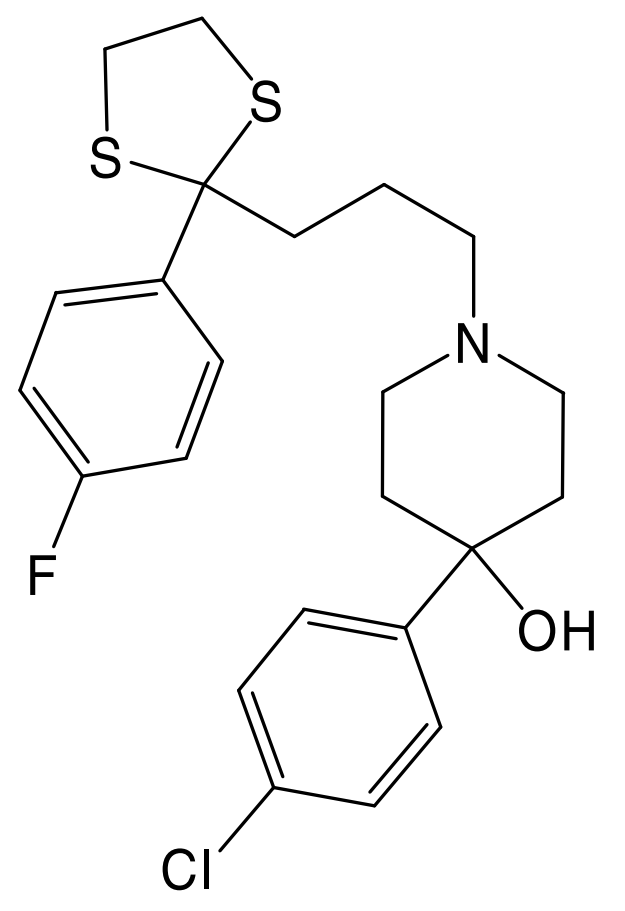

29

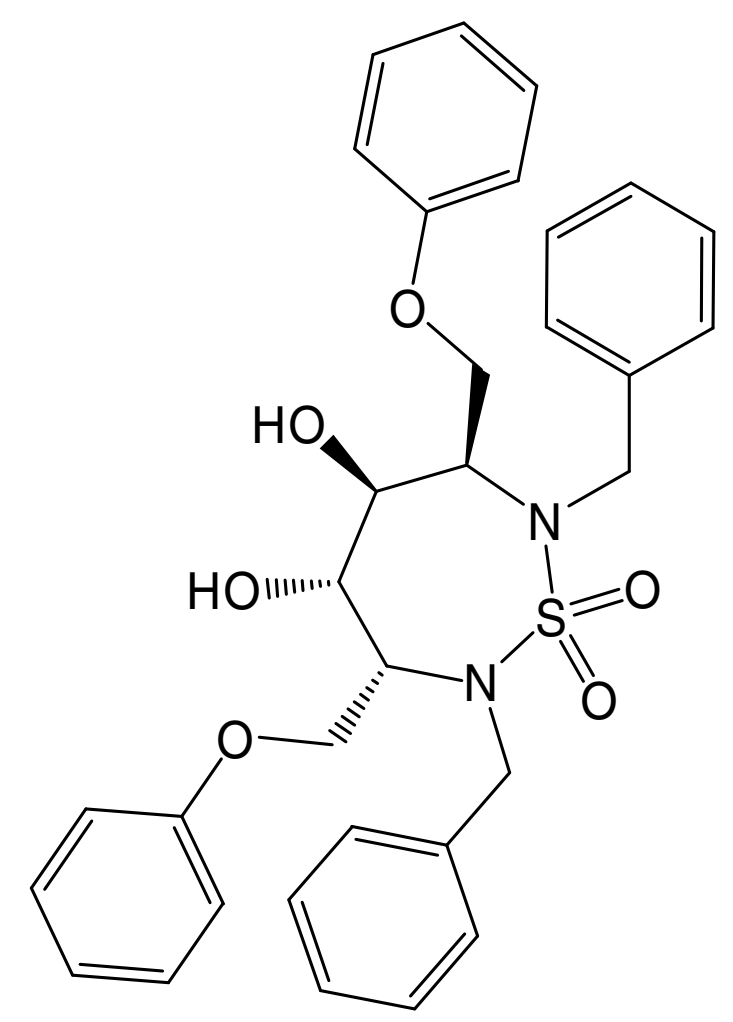

30 


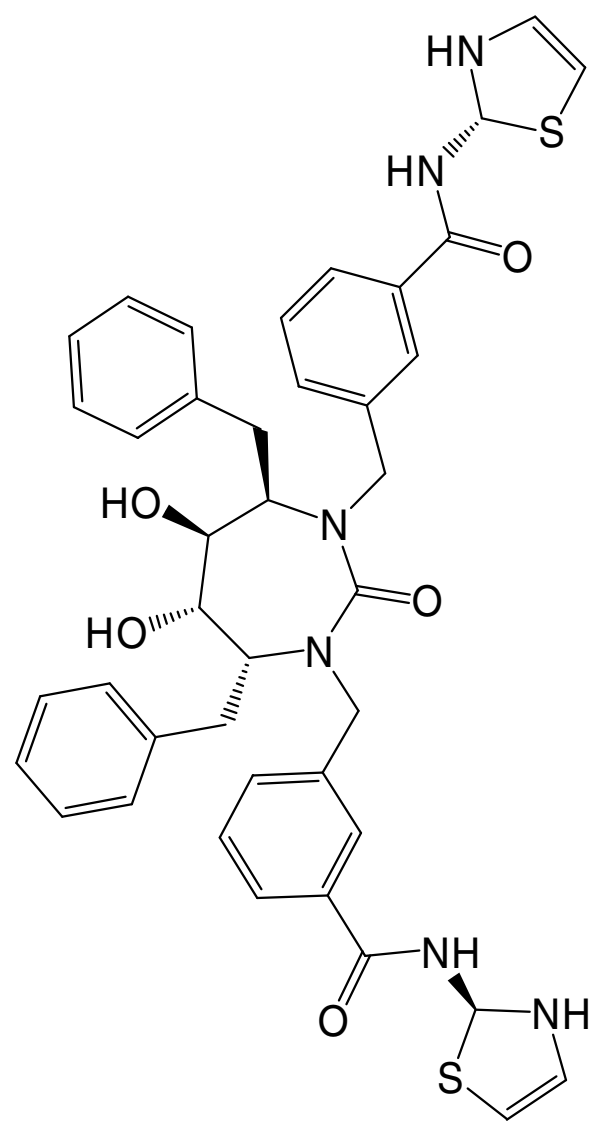

31

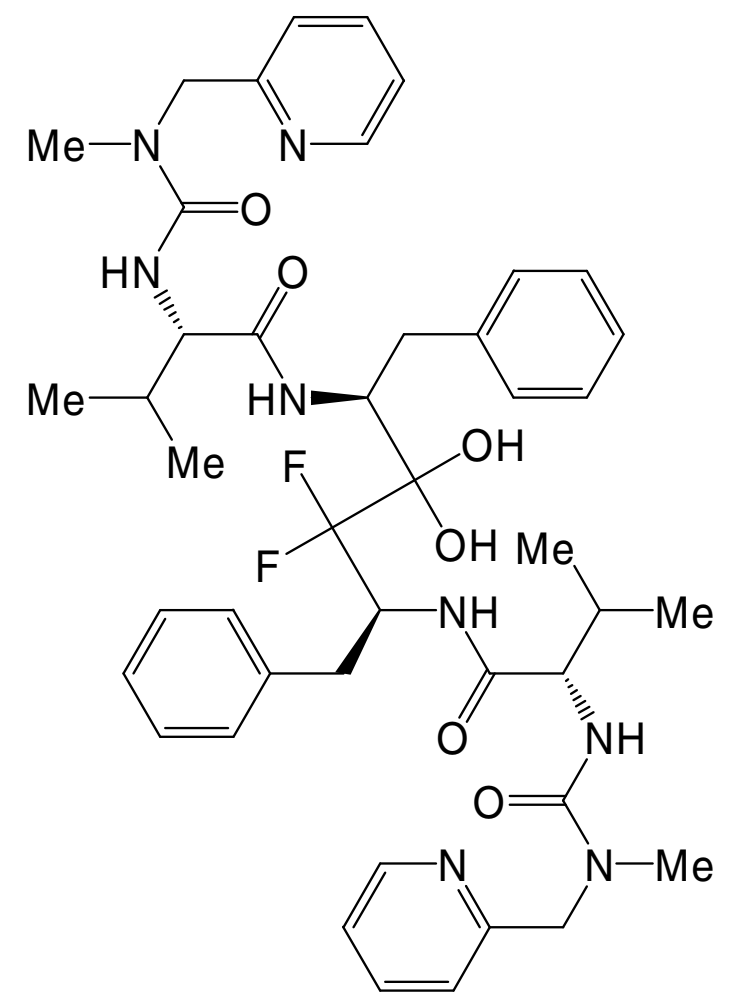

3 2

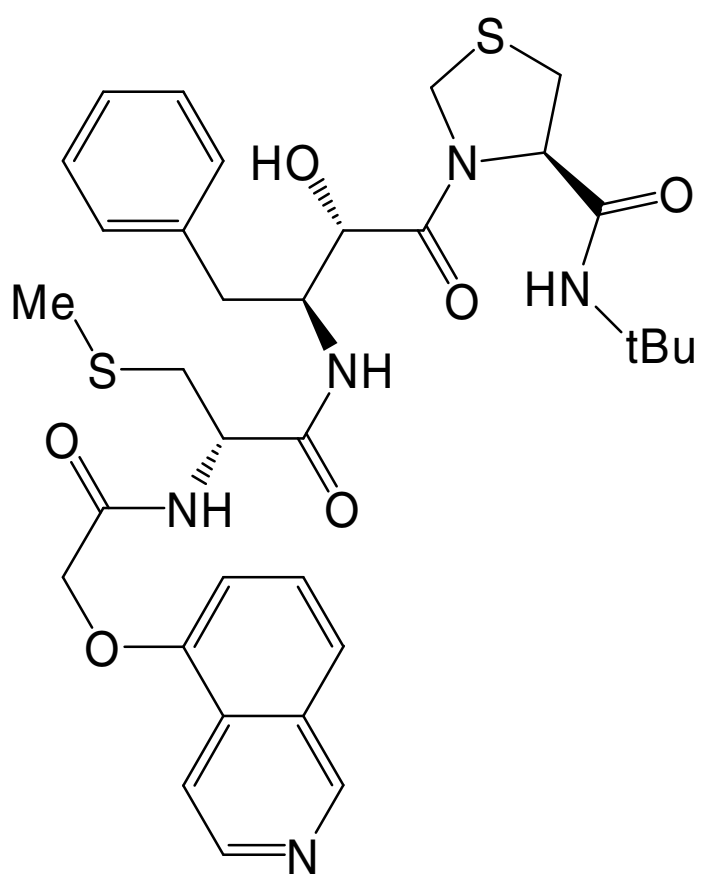



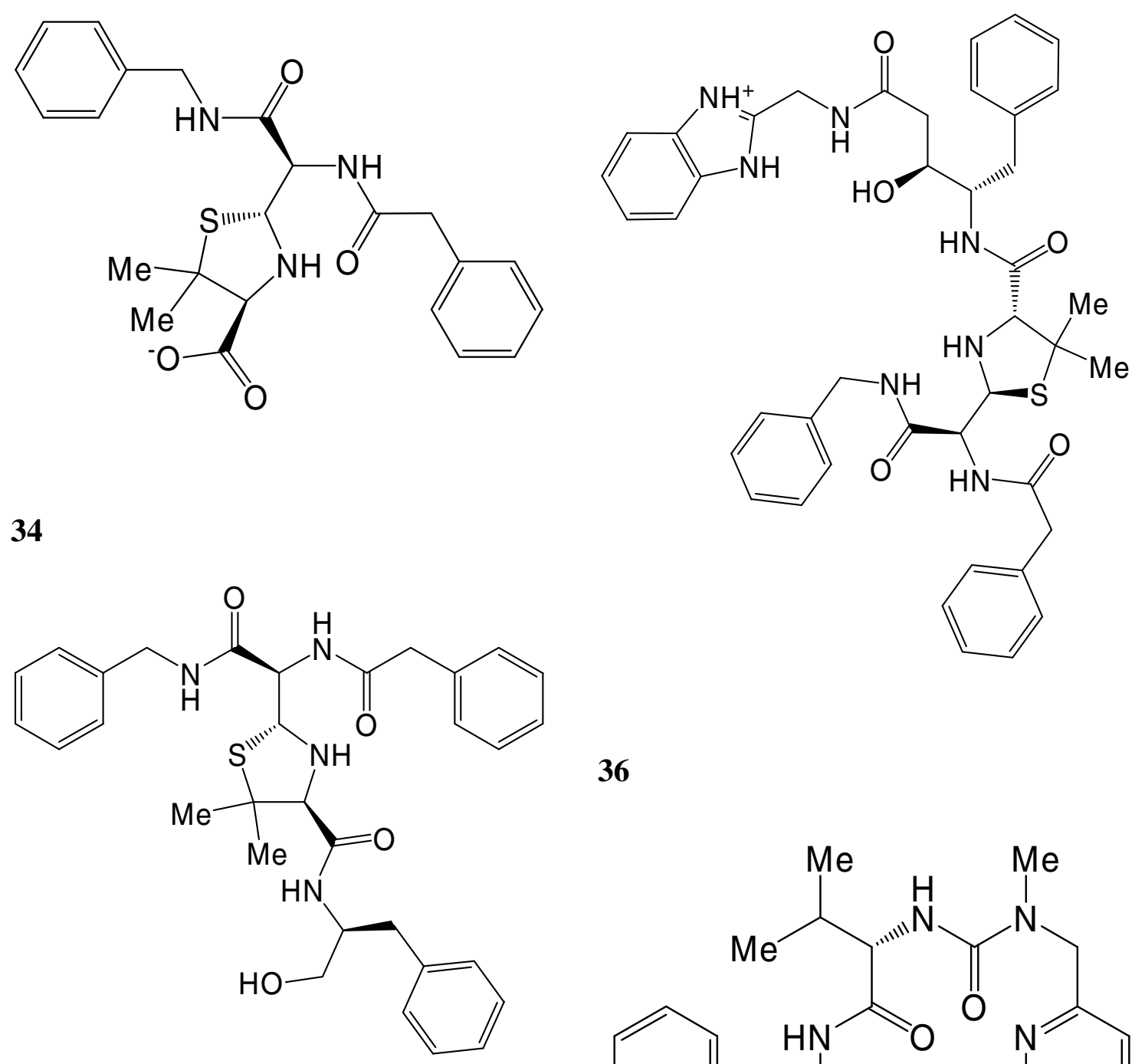

35

36<smiles>CC(C)C(NC(=O)N(C)Cc1ccccn1)C(=O)N[C@H](C(=O)N[C@H](Cc1ccccc1)[C@@H](O)[C@H](O)[C@H](Cc1ccccc1)NC(=O)N(C)Cc1ccccn1)C(C)C</smiles> 

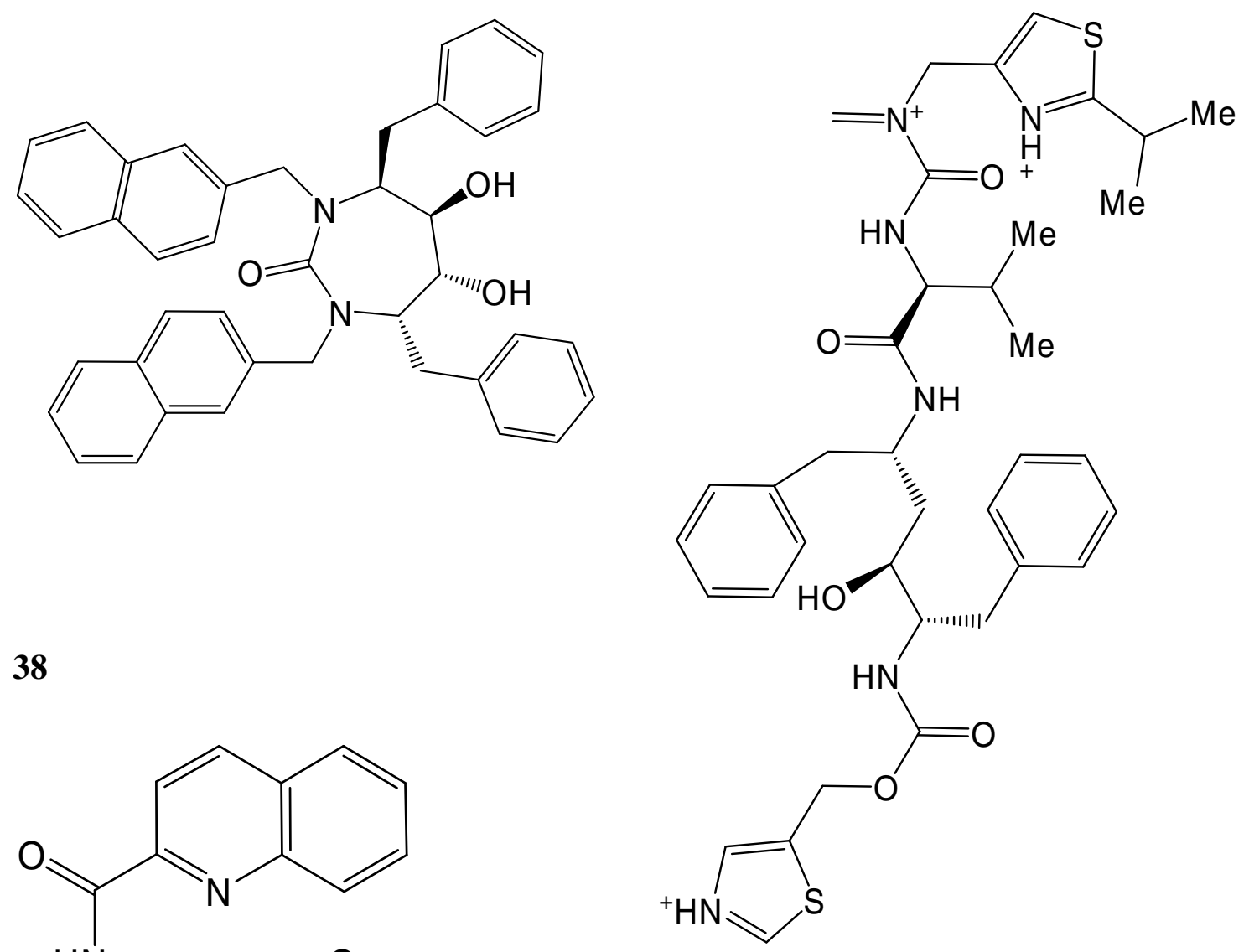

38<smiles>CC(C)(C)NC(=O)[C@@H]1C[C@@H]2CCCC[C@H]2CN1C[C@H](O)[C@H](Cc1ccccc1)NC(=O)[C@H](CC(N)=O)NC(=O)c1ccc2ccccc2n1</smiles>

40

39

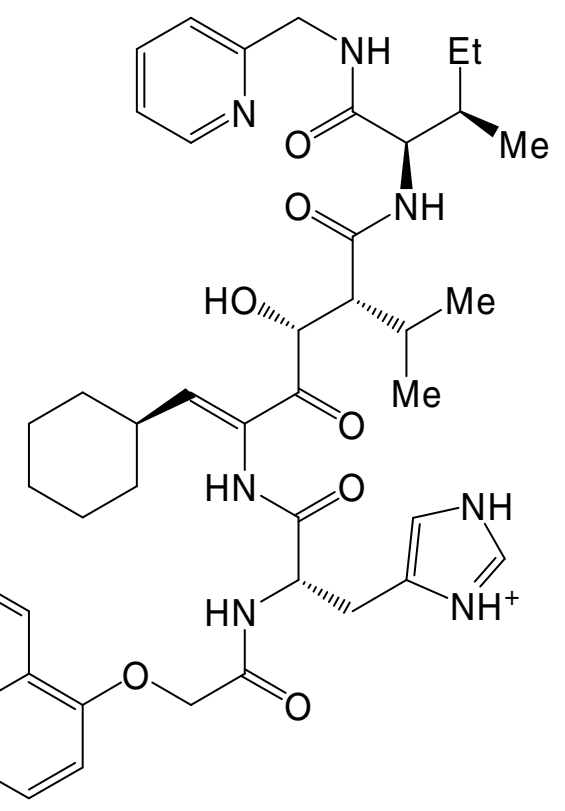

41 

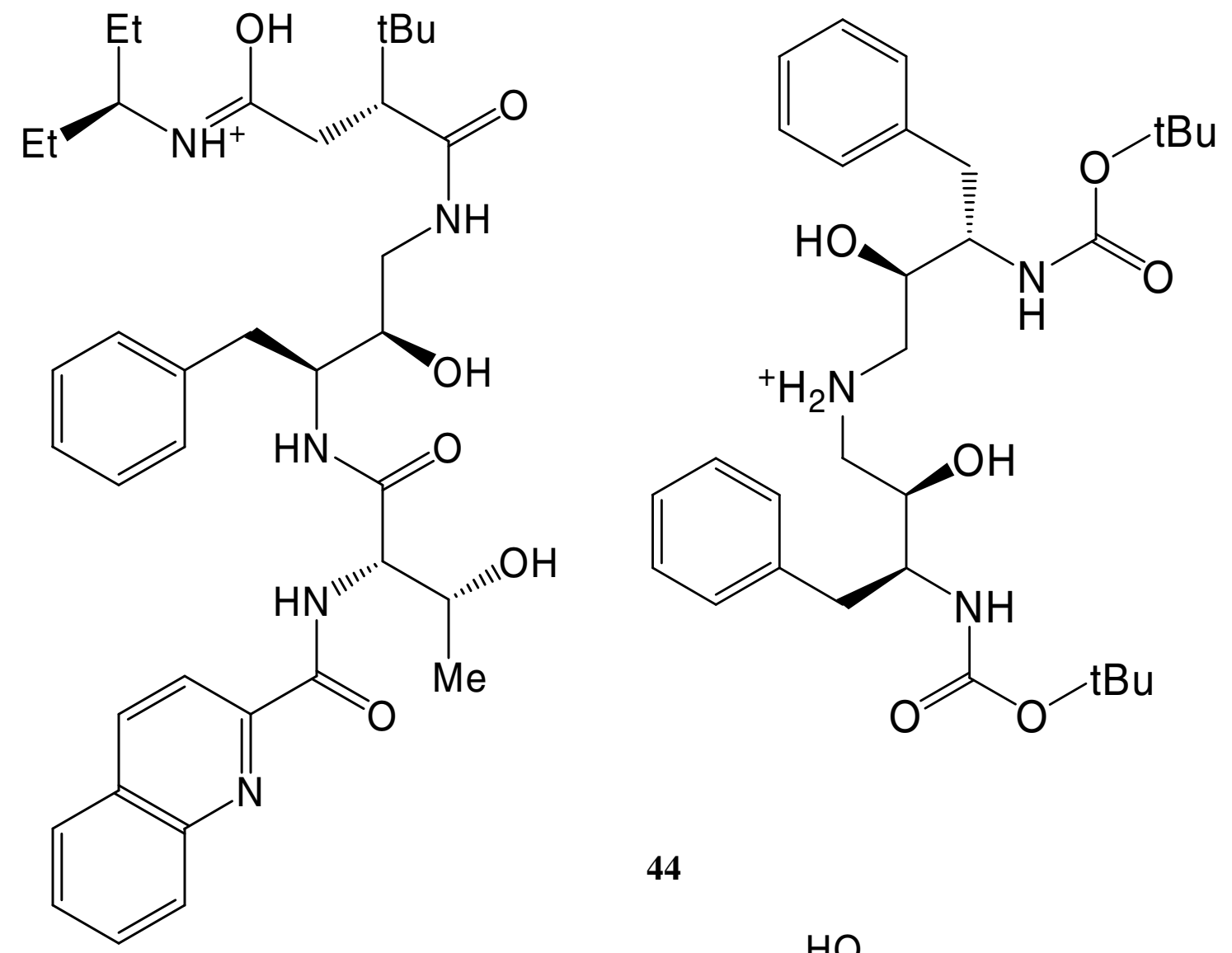

42

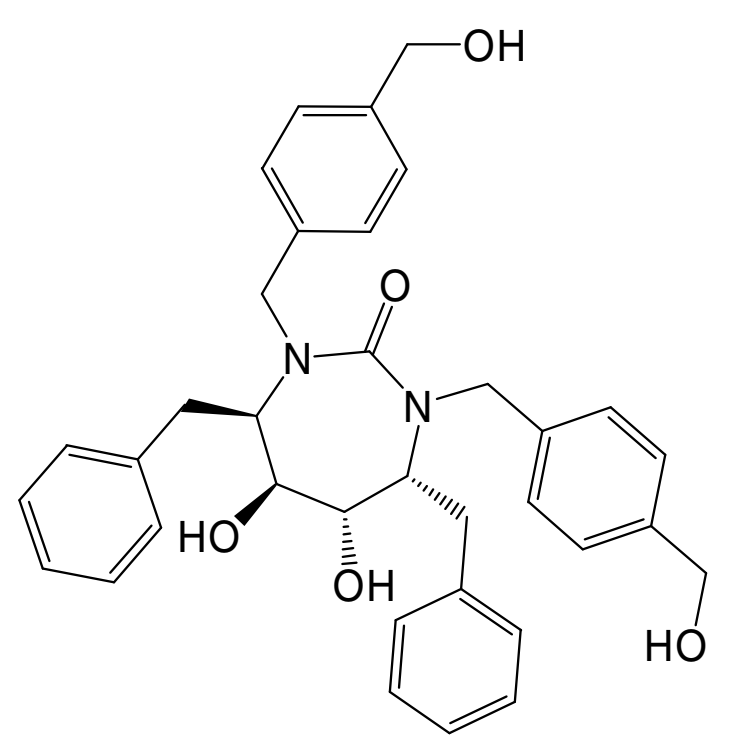

44

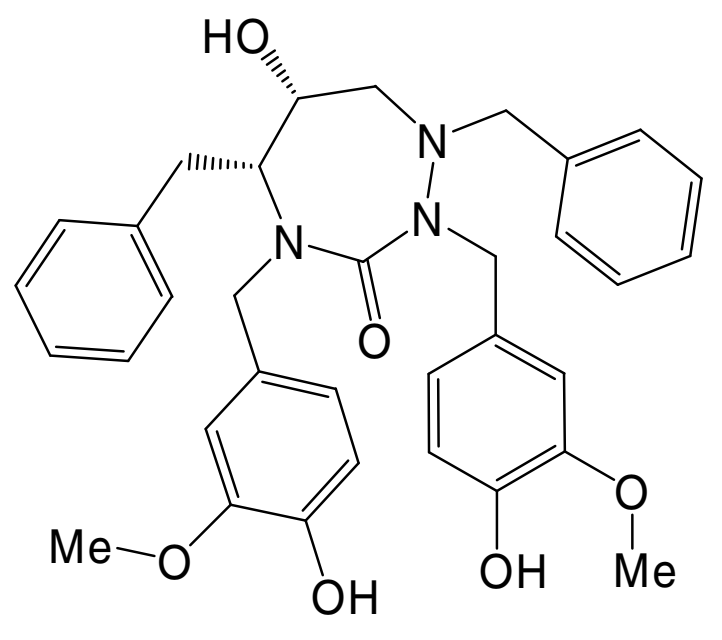

45 
<smiles>CC[C@H](Cc1ccccc1)c1cc(O)c([C@H](c2cccc(NC(=O)CCNC(=O)OCC(C)C)c2)C2CC2)c(=O)o1</smiles>

46
47

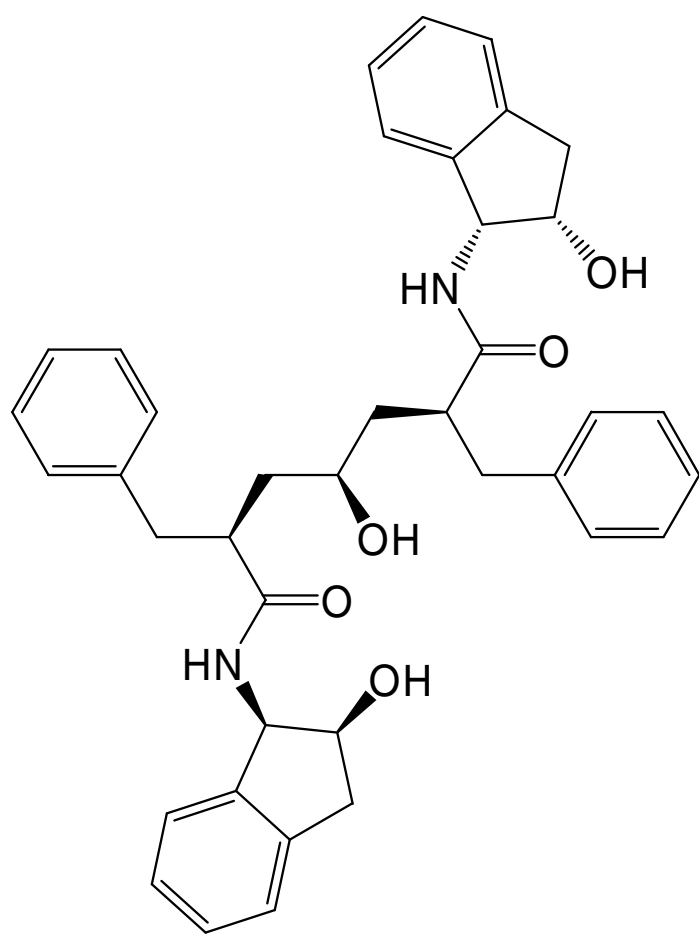

Figure3 Y. Fukunishi, S. Kubota and H. Nakamuidoise reduction method for molecular interaction energ̉y 

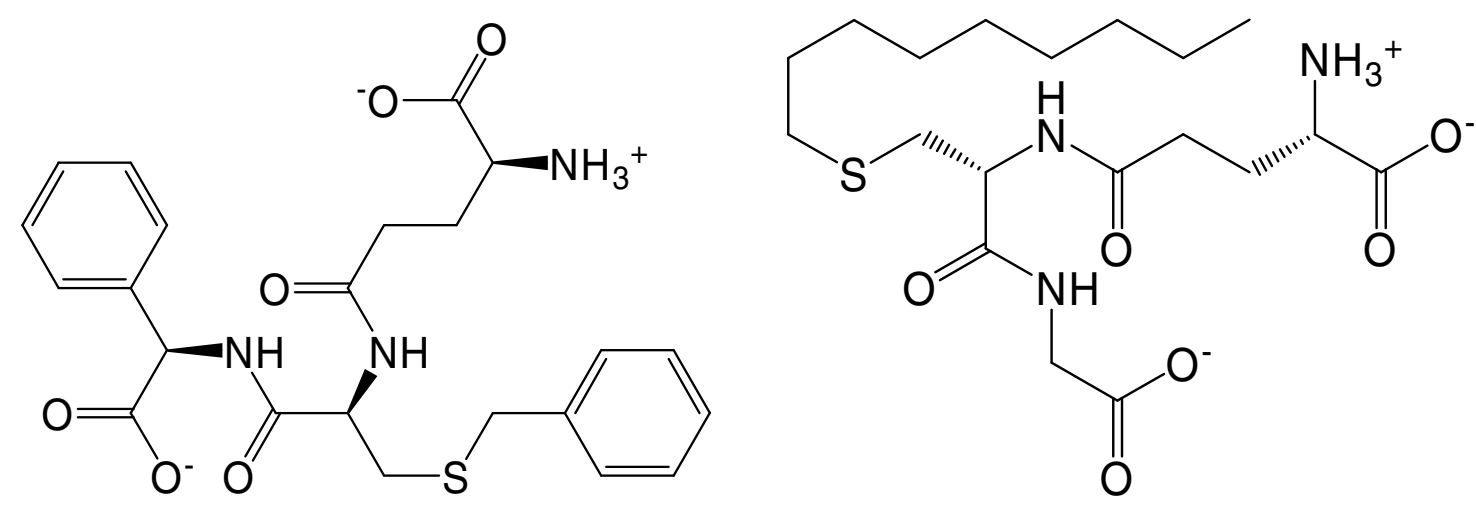

48

50

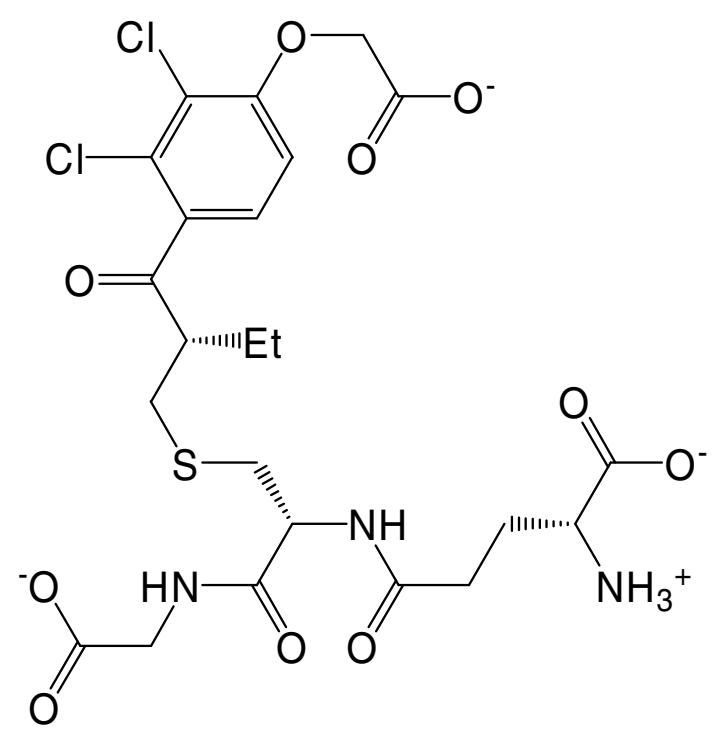

49<smiles>N[C@@H](CCC(=O)N[C@@H](CSc1ccc([N+](=O)[O-])cc1[N+](=O)[O-])C(=O)NCC(=O)[O-])C(=O)[O-]</smiles>

51 

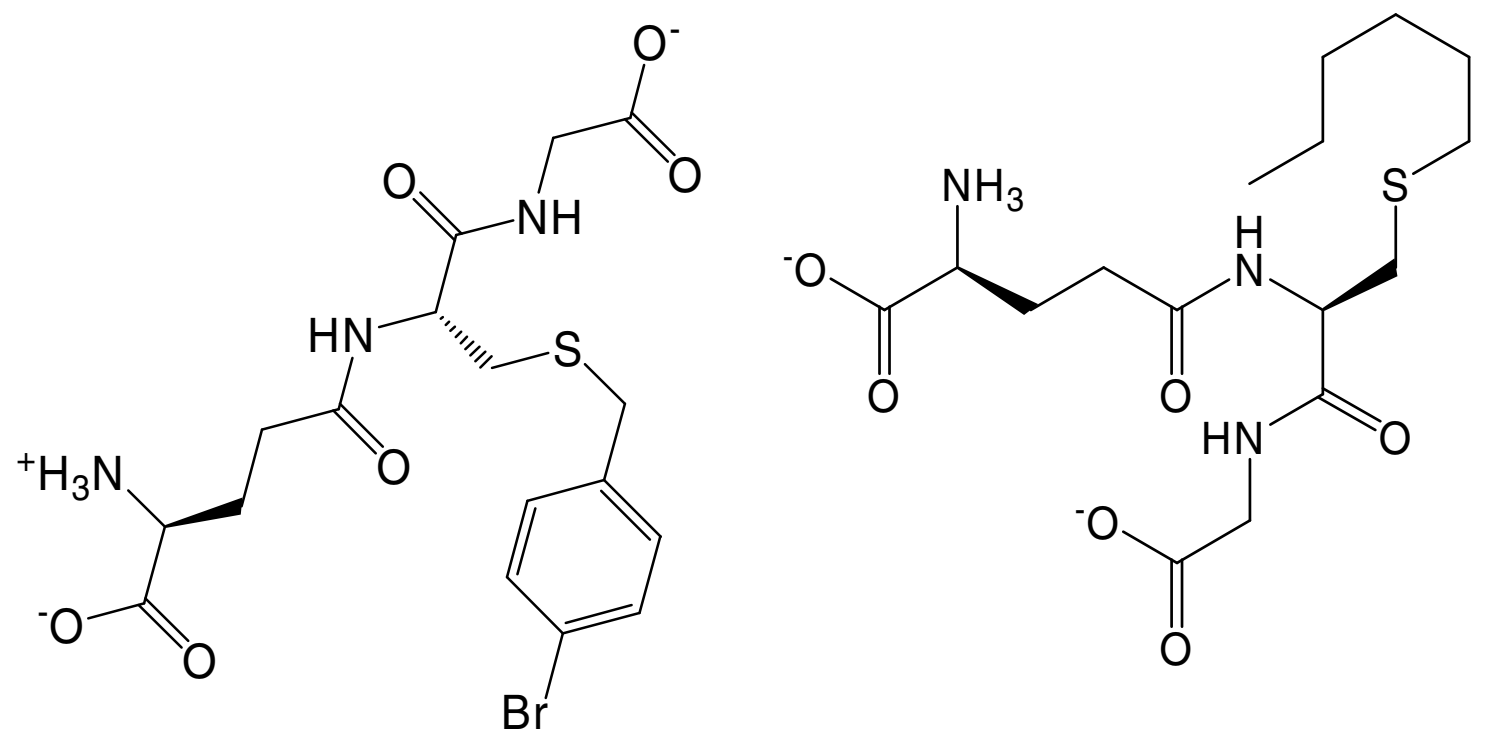

54

52
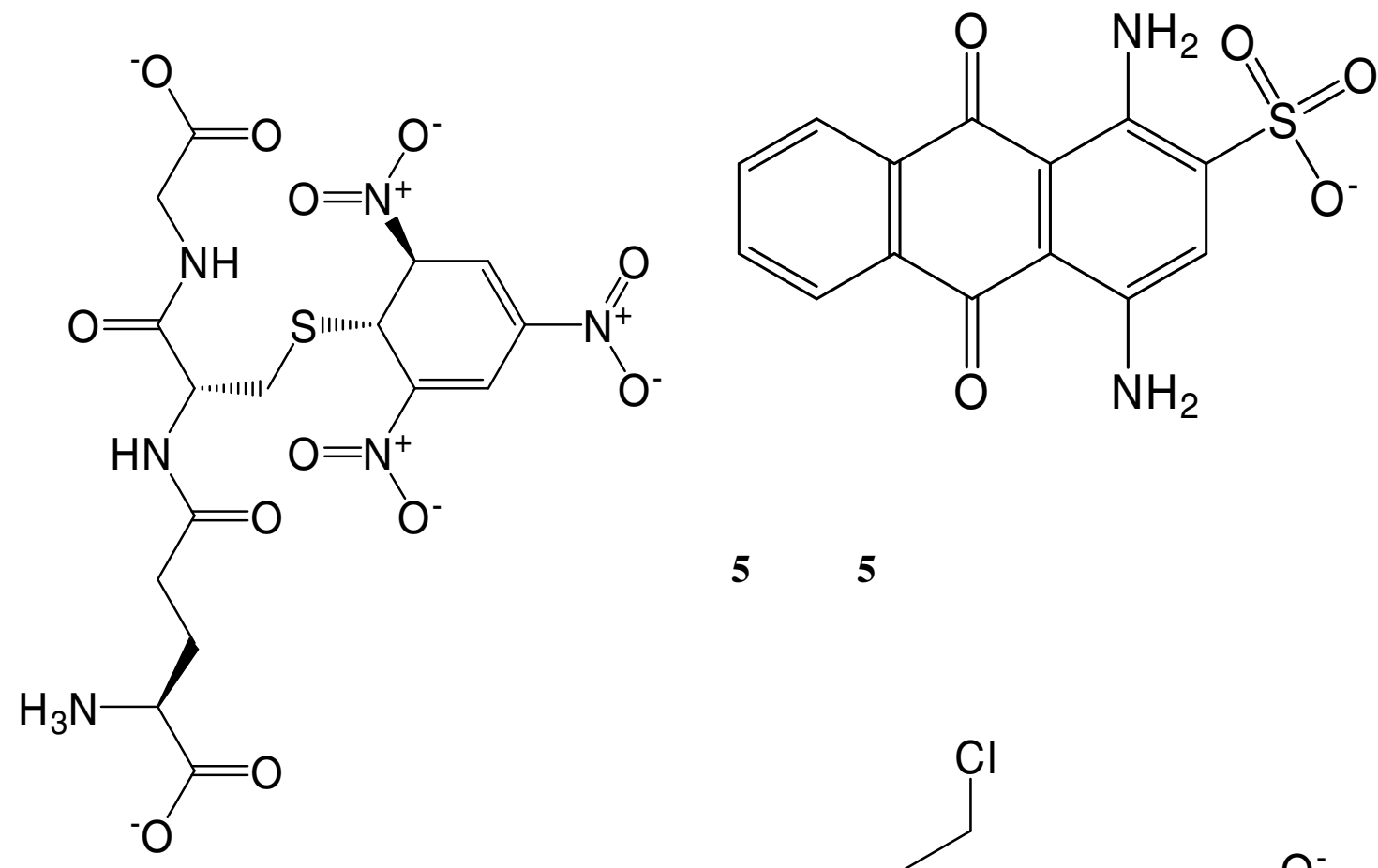

$5 \quad 5$

53<smiles>O=C([O-])CCCc1ccc(N(CCCl)CCCl)cc1</smiles>

56 
<smiles>C=C(CC)C(=O)c1ccc(OCC(=O)[O-])c(Cl)c1Cl</smiles>

57

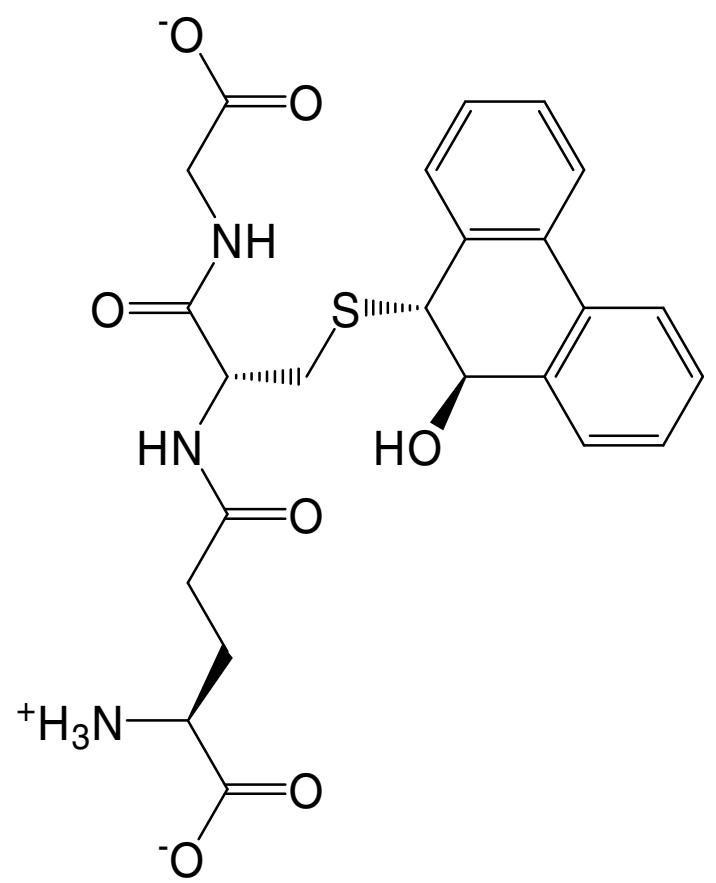

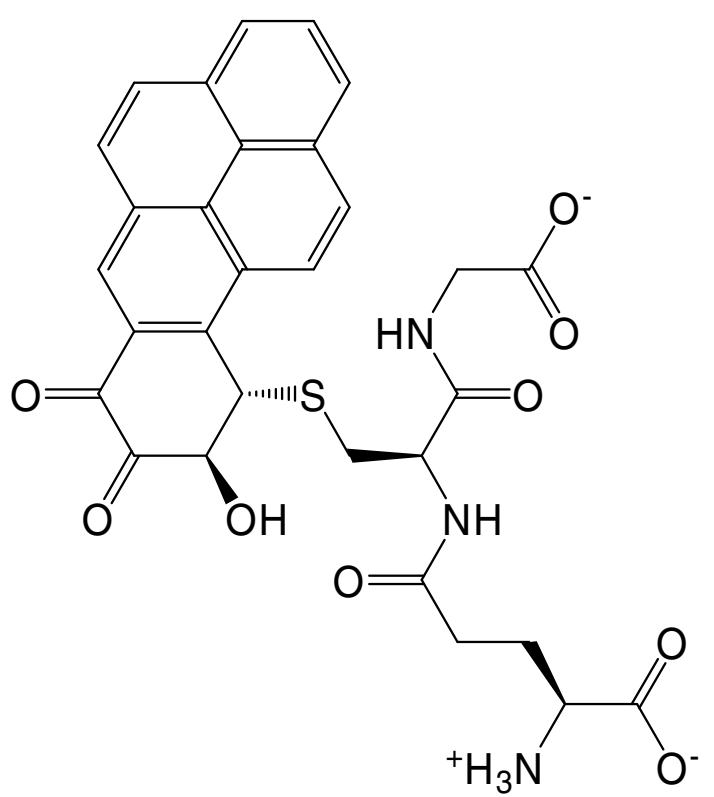

59

58

Figure 4 Y. Fukunishi, S. Kubota and H. Nakamura, "Noise reduction method for molecular interaction energy" 
<smiles>COC(=O)C([NH3+])CC(=O)[O-]</smiles>

60

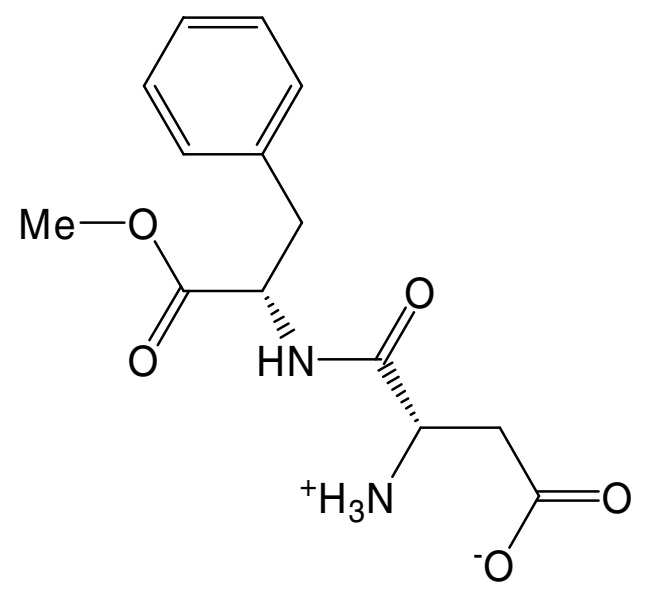

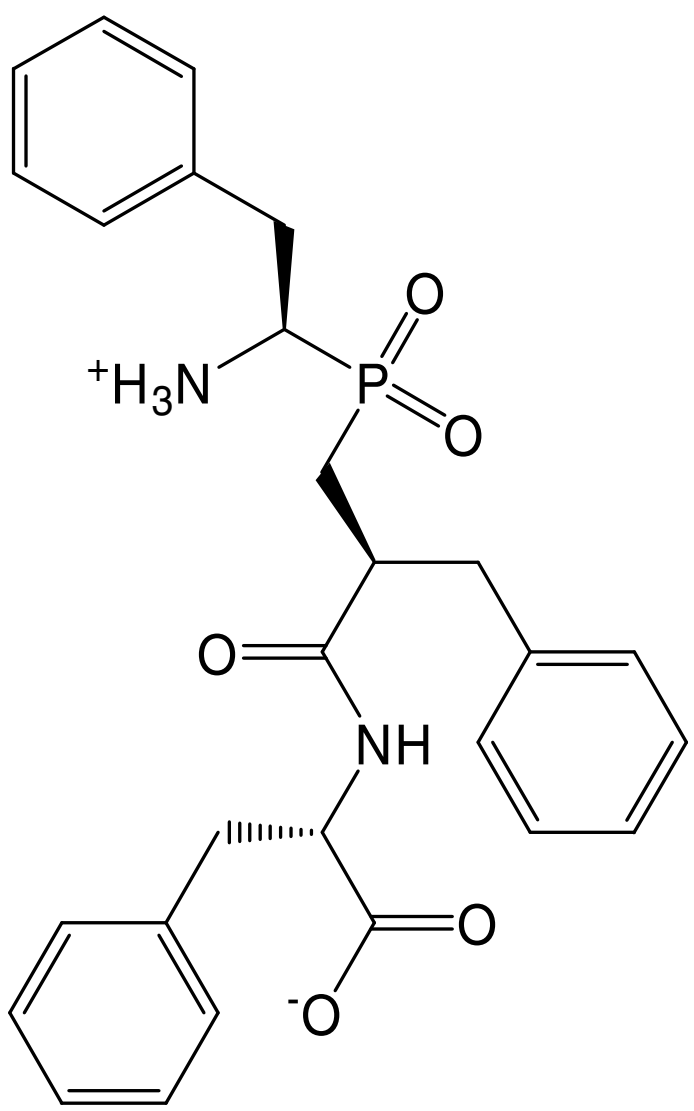

61<smiles>[NH3+][C@@H](Cc1ccccc1)C(=O)[O-]</smiles>

64

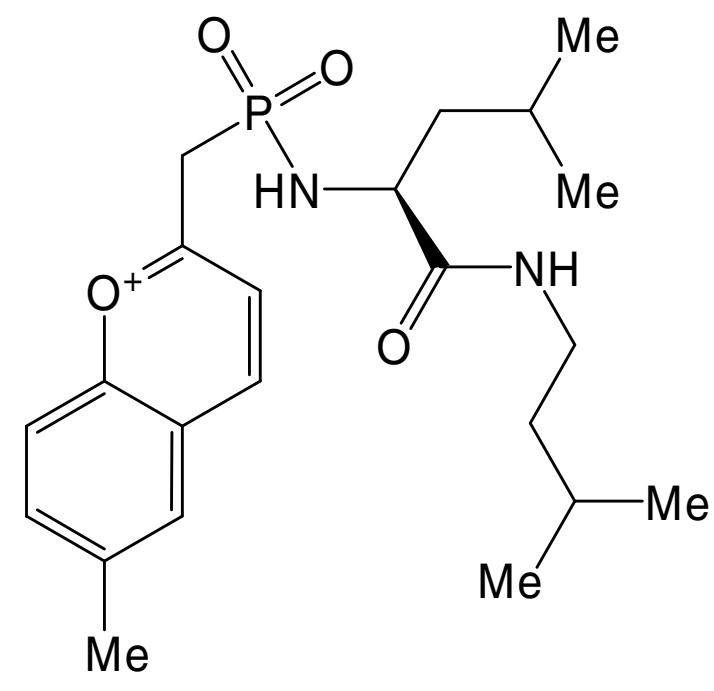

65 

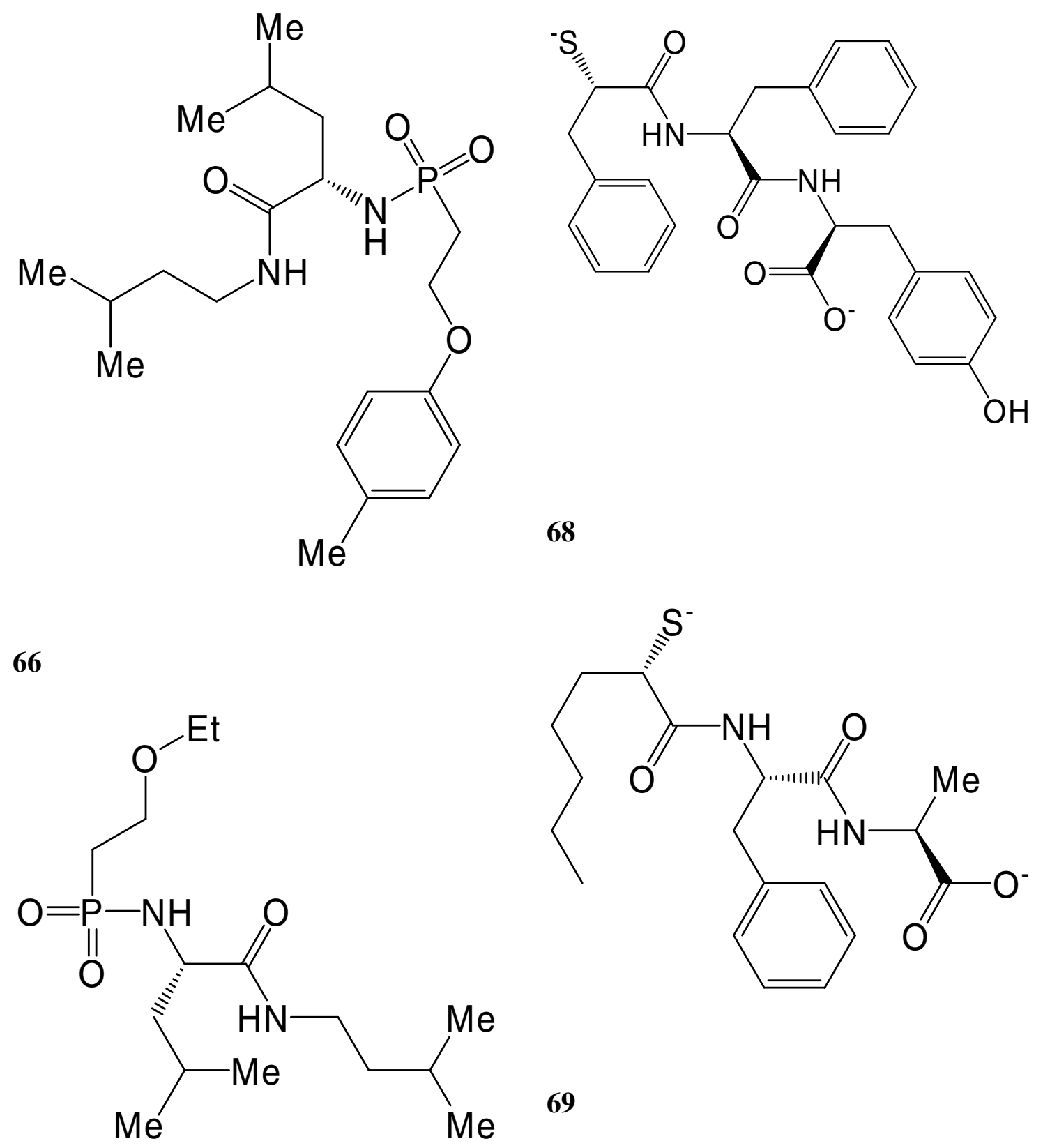

67 

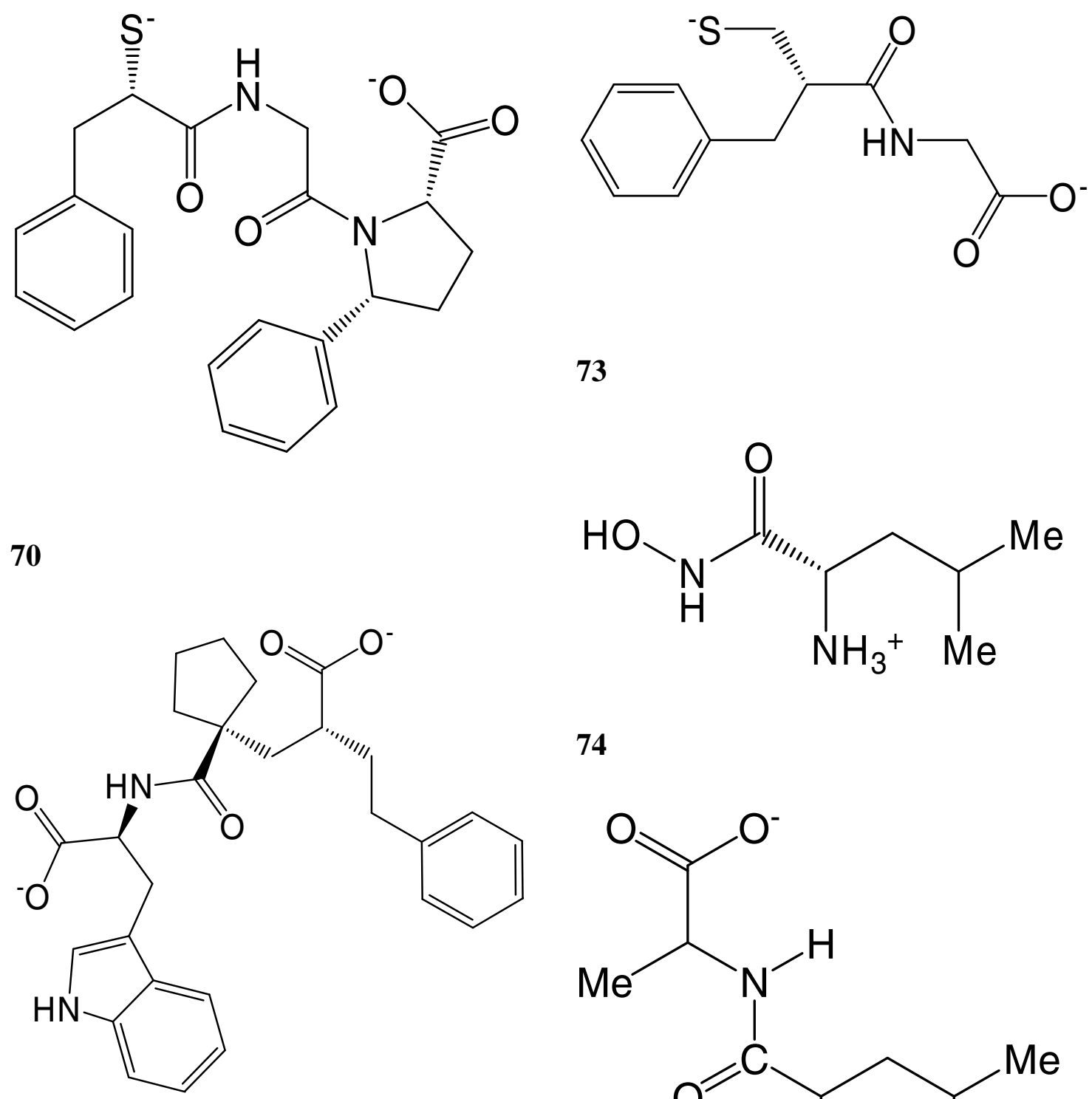

71<smiles>CC(C)C[C@H]([NH3+])C(=O)NO</smiles>

74<smiles>CC(C)CC([NH3+])C(=O)NC(C)C(=O)[O-]</smiles>

75<smiles>O=C([O-])CC(=O)N[C@H](CS)Cc1ccccc1</smiles> 

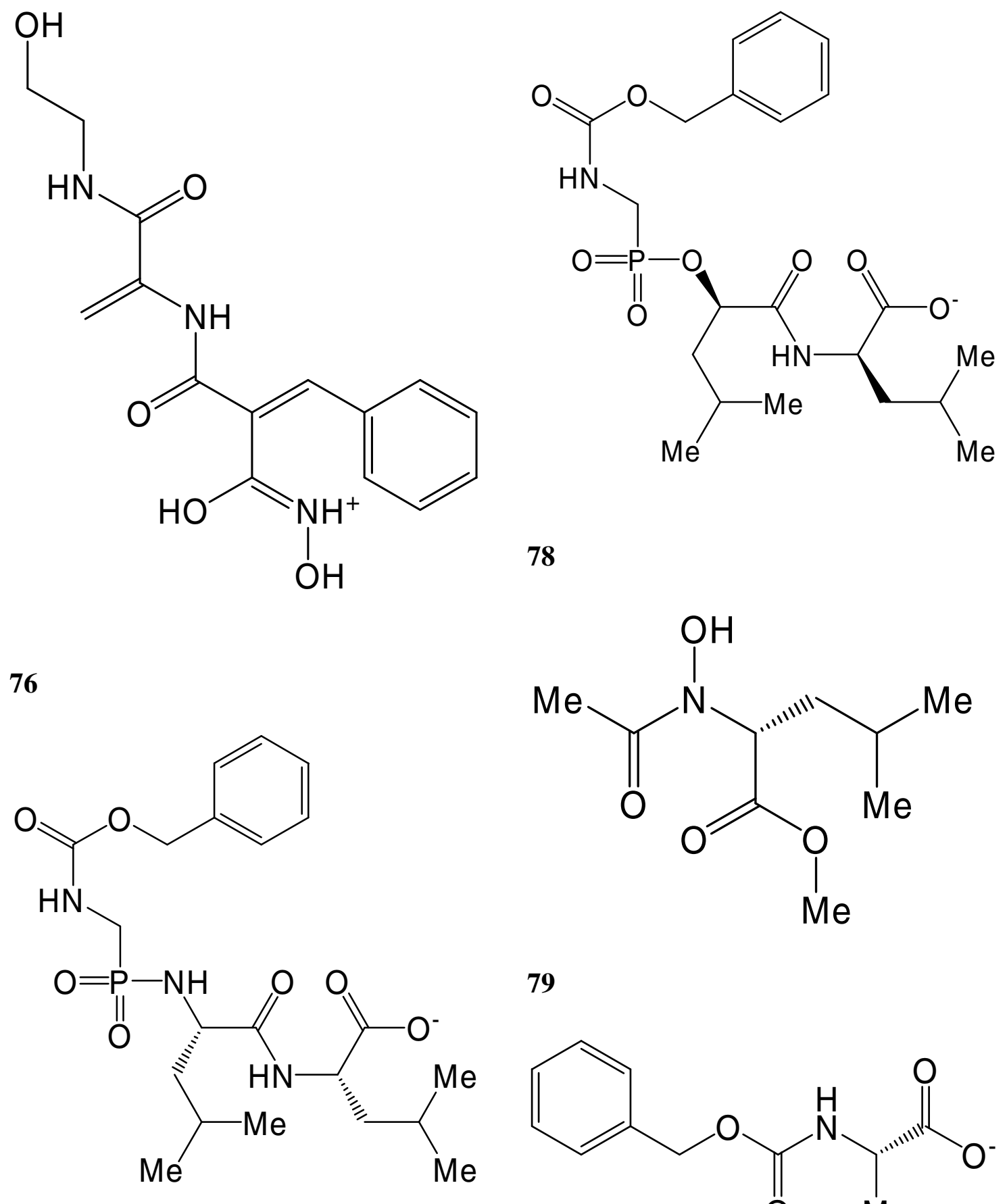

77<smiles>C[C@H](NC(=O)OCc1ccccc1)C(=O)[O-]</smiles> 

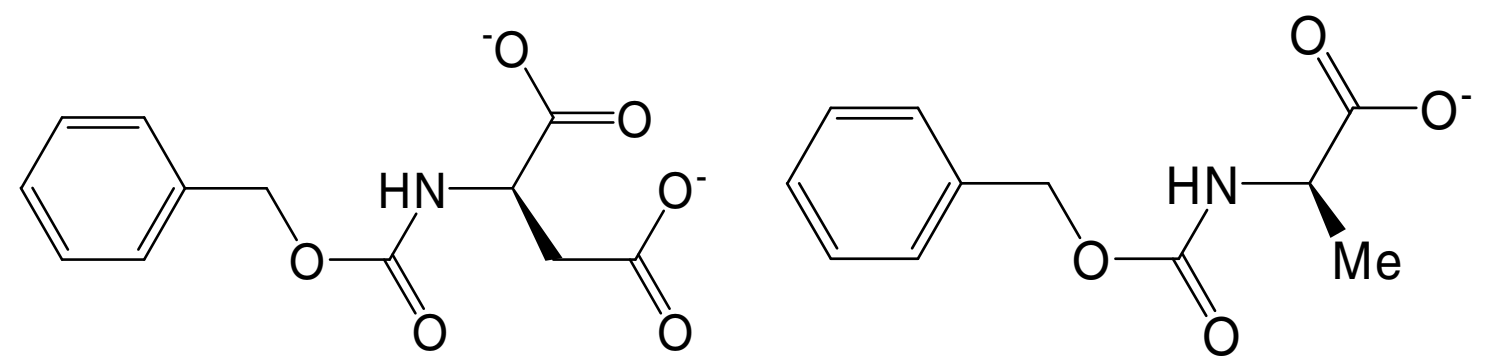

84
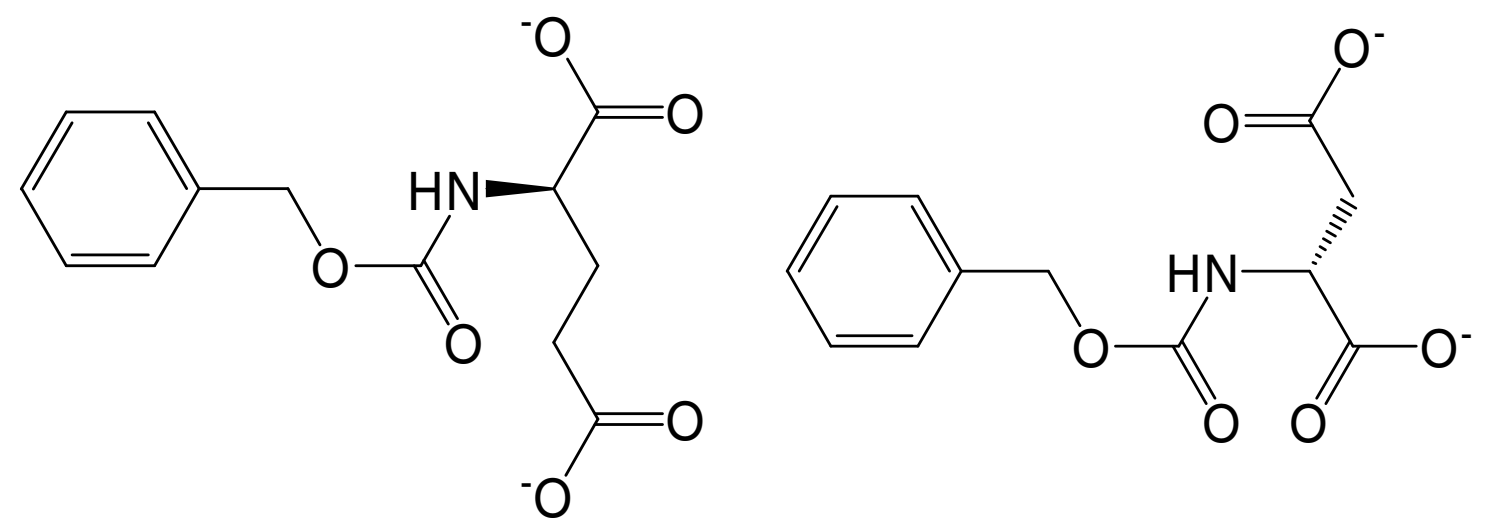

85

82<smiles>C[C@@H](O)[C@H](NC(=O)OCc1ccccc1)C(=O)[O-]</smiles>

83

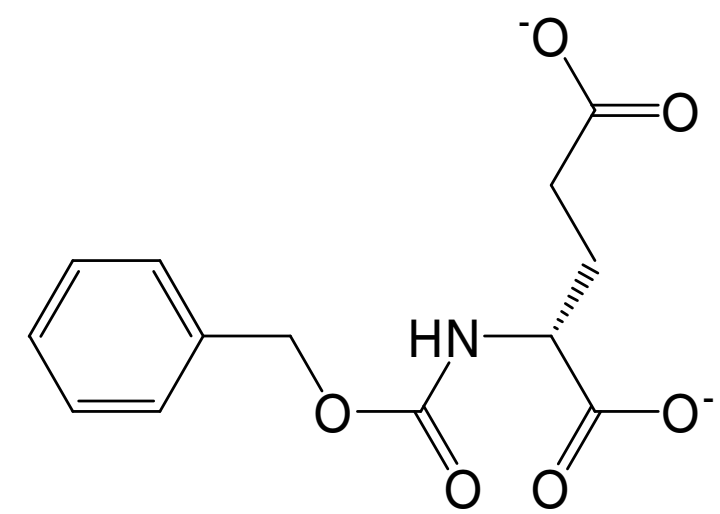

86 


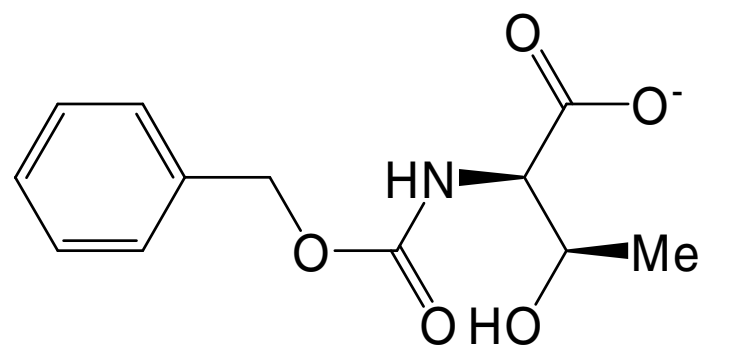

87

Figure 5 Y. Fukunishi, S. Kubota and H. Nakamura, "Noise reduction method for molecular interaction energy" 
